\title{
Interações biofísicas em espécies arbóreas potencialmente acumuladoras de fósforo: diversidade de irradiância e de comportamento hídrico
}

\author{
Patricia Chaves de OLIVEIRA ${ }^{1}$, Cláudio José Reis de CARVALHO²
}

\begin{abstract}
RESUMO
O entendimento das interaçōes biofísicas entre espécies acumuladoras de fósforo, como Neea macrophylla e Cecropia palmata com a radiação e a água disponível em florestas secundárias no Nordeste Paraense torna-se fundamental para a composição de Sistemas Agroflorestais e Florestas Enriquecidas. O objetivo desta pesquisa foi o de identificar o quanto atravessava de radiação (PAR, BAR e PhyAR) pelas copas das espécies estudadas levando em consideração a densidade de agregação das árvores e a distância vertical a partir do solo, bem como o comportamento hídrico através da determinação dos potenciais hídricos (potencial antes do amanhecer e após meio-dia) em diferentes períodos climáticos. Os resultados mostraram que Cecropia palmata obteve as maiores irradiâncias independente da agregação das árvores sugerindo melhores condições aos processos fotossintéticos e morfogênicos às espécies que vierem a se localizar sob influência de suas copas. Quanto ao comportamento hídrico observou-se uma estratificação bem nítida entre as três espécies, onde Cecropia palmata foi a espécie que desenvolveu os maiores Potenciais Hídricos após o meio-dia sinalizando maior tolerância a períodos secos e por conseguinte se mantendo mais estável a condições de turgidez, o que é essencial aos processos de fotossíntese enquanto Casearia arborea pode ser considerada a mais sensível pois atingiu Potenciais Hídricos $(\psi)$ muito negativos. O efeito do período climático sobre os Potencias Hídricos determinou uma redução destes nos meses com menor precipitação pluviométrica para todas as espécies.
\end{abstract}

PALAVRAS-CHAVE: Potencial hídrico, Radição Fotossinteticamente ativa (PAR), Radiação Ativa ao azul (BAR), Sistemas agroflorestais.

\section{Biophysic interaction in potentially accumulative phosphorus wood species: irradiance diversity and hydric behavior}

\begin{abstract}
The agreement of biophysic interaction between phosphorus accumulative species, such as Neea macrophylla and Cecropia palmata with radiation and water available in secondary forests in the Northeast of Pará, becomes essential for the composition of Agroforest Systems and enriched Forests. The objective of this research was to identify how much radiation (PAR, BAR and PhyAR) crossed over to the canopies of the species studied, taking into consideration the tree aggregation density and distance from the soil, as well as the water behavior by determining the Water Potential (predawn and midday) in different climatic periods. The results showed that Cecropia palmata had the highest irradiances, independent of tree aggregation, suggesting better conditions for photosynthetic and morphogenic processes of the species that comes under the influence of its canopies. In respect to water behavior, a clear stratification between three species was observed, where Cecropia palmata was the species that developed the highest water potential midday, signaling fantastic tolerance to dry periods and consequently maintaining a more stable turgidity condition, which is essential to the photosynthetic process, while Casearia arborea can be considered the more sensitive, for it reached a very negative Water Potencial $(\psi)$. The effect of the climatic period on the Water Potential determined their reduction for all species in the months with less pluviometric precipitation-
\end{abstract}

KEY WORDS: water potential, Photosynthetically Active Radiation (PAR), Blue Active Radiation (BAR), Agroforest Systems

1 Dra. em Ciencias Agrárias, professora adjunta da UNIVERSIDADE FEDERAL DO PARÁ/Campus de Santarém, pchaves@ufpa.br.

2 Fisiologista de plantas, EMBRAPA/CPATU, carvalho@embrapa.cpatu.br 


\section{INTRODUÇÃO}

O dossel de uma determinada floresta pode influenciar a quantidade e a qualidade de radiação que passa através dele. Dessa forma, tanto a Radiação Fotossinteticamente Ativa (PAR), Radiação Ativa ao Azul (BAR) quanto a Radiação Ativa ao Fitocromo (PhAR) podem variar em função da arquitetura da copa das espécies. De acordo com Sá et al (1999) em vegetações secundárias enriquecidas no Nordeste Paraense, Acacia mangium Willd foi a espécie que apresentou a menor PAR abaixo de suas copas, sugerindo que sua associação com espécies tolerantes a baixos níveis deste tipo de radiação devam se adaptar melhor. Em espécies arbóreas potencialmente acumuladoras de $\mathrm{P}$ pouco se conhece acerca de suas interações biofísicas sobretudo quanto a irradiância espectral que atravessa o dossel destas espécies.

A altura dentro de um perfil vertical em floresta Tropical Úmida na Amazônia Central exerceu influência sobre algumas respostas fotossintéticas como observou Carswell et al (2000). A taxa máxima de transportes de elétrons $\left(\mathrm{J}_{\mathrm{MAX}}\right)$ e a taxa máxima de carboxilação pela rubisco $\left(\mathrm{V}_{\mathrm{CMAX}}\right)$ aumentaram significativamente com o aumento da altura dentro da floresta, onde os valores máximos obtidos para $\mathrm{J}_{\mathrm{MAX}}$ foram de 103,9 $\mu$ mol.m $\mathrm{m}^{-2} \cdot \mathrm{s}^{-1}$ a $20 \mathrm{~m}$ comparado com 35,8 $\mu \mathrm{mol} \cdot \mathrm{m}^{-2} . \mathrm{s}^{-1}$ ao nível do solo e para $\mathrm{V}_{\text {CMAX }} 42,8 \mu \mathrm{mol} . \mathrm{m}^{-2} .^{-1}$ a $24 \mathrm{~m}$ e $20,5 \mu \mathrm{mol} . \mathrm{m}$ ${ }^{2} . s^{-1}$ ao nível do solo. Os autores ainda observaram correlação positiva entre a capacidade fotossintética e a concentração de nitrogênio nas folhas, as quais aumentavam com a altura dentro do dossel o que pode ser explicado através da redução da área foliar específica (SLA). Dessa forma, dependendo do estágio de desenvolvimento de florestas secundárias as diferenças de respostas fotossintéticas entre os estratos podem ser maiores ou não; é de se esperar que durante a fase inicial de regeneração da capoeira o gradiente de respostas fotossintéticas seja menor entre espécies pioneiras, aumentando ao longo do processo de sucessão ecológico.

A distribuição de luz ao longo do perfil vertical dos dosséis, pode determinar alteraçôes na distribuição do ângulo foliar e na razão de bifurcação do caule, como observado em indivíduos de Quercus, nos quais o ângulo foliar variou de $1,6^{\circ}$ no alto da copa a $3,2^{\circ}$ nos ramos mais baixos (Kull et al ,1999). Da mesma forma, a razão de bifurcação dos caules e o número de folhas por ramo diminuíram com a redução da densidade do fluxo de fótons (PFD), sugerindo que este é um parâmetro que influencia a arquitetura das copas das espécies e por conseguinte o particionamento de luz dentro e abaixo das plantas. Relacionar a arquitetura da copa de espécies potencialmente acumuladoras de $\mathrm{P}$ com as variaçōes na PFD ao longo das estaçóes seca e chuvosa, indicaria a sensibilidade ou não da espécie a este fator, pois uma redução no número de folhas por ramo, por exemplo, em situaçōes de baixa
PFD, levaria a um menor acúmulo de fitomassa aérea e por conseguinte menor quantidade de litter.

A variação da precipitação no sistema, ou seja nas florestas secundárias, parece ser determinante no comportamento hídrico de espécies comuns nestes ambientes. A capacidade de estoque de água que cada espécie apresenta em sistema de regeneração natural das capoeiras certamente varia com características morfo-anatômico e metabólicas. Contudo, o tamanho da árvore parece relacionar-se diretamente com o tempo de fluxo máximo no xilema conforme Meinzer et al (2001). Segundo os autores, em 27 espécies que co-ocorrem em floresta tropical a densidade do fluxo máximo do xilema medido na base dos caules variou com o tempo em função do tamanho das árvores, sendo que nas maiores ocorreu às 14:00 $\mathrm{h}$ e nas menores às 11:30 h. Além disso, os déficits de pressão de vapor atmosférico influenciam de forma decisiva os fluxos de água das plantas para o ambiente principalmente nas espécies menos tolerantes.

Pouco ainda se sabe sobre os fluxos de água em espécies potencialmente acumuladoras de P. Segundo Sellin (2001), a densidade do fluxo de fótons (PFD) influenciou as respostas estomáticas de folhas posicionadas na luz e na sombra de Picea abies com alta condutância máxima diária de vapor de água encontrada nas primeiras. Além disso, a idade das plantas foi outro parâmetro determinante na velocidade dos movimentos estomáticos, sendo muito rápidas em plantas mais velhas. A riqueza florística e o potencial econômico que determinadas espécies da Amazônia têm como a Copaifera ssp na produção de óleo de copaíba (Rigamonte et al, 2005) entre outras nos alerta para a elucidação das estratégias que espécies nativas possam desenvolver quanto ao estresse hídrico. A plasticidade estomática das espécies passa a ser uma característica importante na competição por água dentro do sistema capoeira, pois indica uma capacidade de se adaptar às variaçôes de oferta do recurso, bem como o ajustamento osmótico (Carvalho, 2005).

Os objetivos desta pesquisa foram o de quantificar a Radiação Fotossintéticamente Ativa (PAR), Radiação Ativa ao Azul (BAR) e Radiação Ativa ao Fitocromo (PhyAR) que passavam através das copas de algumas árvores das espécies acumuladoras de P, Neea macrohylla e Cecropia palmata, a fim de se avaliar o potencial de associação destas espécies em sistemas agroflorestais. A disponibilização de luz bem como o perfil do comportamento hídrico destas plantas ao longo de diferentes condiçōes de precipitação propiciará a identificação de graus de tolerância de espécies acumuladoras de P à déficits hídricos.

\section{MATERIAL E MÉTODOS}

As espécies estudadas, Neea macrophylla, Cecropia palmata e Casearia arborea encontravam-se em floresta secundária (sítio 
I) aproximadamente com 6 anos de regeneração adjacente a uma outra floresta mais antiga (20 anos) localizada na Fazenda-Escola da Universidade Federal Rural da Amazônia, no Município de Igarapé-Açú à Nordeste do Estado do Pará. As árvores estavam distribuidas ao acaso e distanciadas aproximadamente 10 metros. O solo da área se caracterizava pela baixa fertilidade, com $2 \mathrm{mg}$ de $\mathrm{P} / \mathrm{dm}^{-3}$ solo, baixas porcentagens de $\mathrm{N}$, cerca de $0,03 \%, \mathrm{PH}$ ácido, na faixa de 4,5 a 5,0 e concentraçóes expressivas de $\mathrm{Al}$, em torno de 0,5 cmol dm ${ }^{-3}$ solo.

A determinação da Irradiância Espectral foi descrita acima e abaixo das copas das espécies estudadas durante a estação seca e expressas $\mathrm{em} \mathrm{W} / \mathrm{m}^{2} / \mathrm{nm}$. O aparelho utilizado para determinação da Irradiância Espectral foi um espectroradiômetro portátil (Li-1800, Li-Cor Inc., Lincoln, Nebraska, USA) (Pearcy, 1989). A quantificação da radiação foi realizada por faixas de comprimento de onda (nm); especificamente Radiação Fotossinteticamente Ativa (PAR), entre 400-700 nm (McCree, 1981); Radiação Ativa ao Azul (BAR), entre 400 e 500 nm (Woodward, 1983) e Radiação Ativa ao Fitocromo (PHYTAR) correspondente à razão 655-665 nm/ 725-735 nm.

A determinação do Potencial Hídrico foliar foi medido em ramos com folhas fisiologicamente maduras (funcionais) nas 3 espécies estudadas, às 5:30 (potencial de base) e às 12:00 horas (após meio-dia) em dias típicos do período seco, chuvoso, de transição e de estiagem com uma bomba de pressão.

$\mathrm{A}$ análise Estatística quanto às Radiaçôes PAR, BAR e PhyAR que atravessavam as copas de Neea macrophylla e Cecropia palmata foi feita através de Análise Multivariada a partir da aplicação do Teste de Hotteling. Quanto à avaliação dos Potenciais Hídricos esta inicialmente foi realizada através da construção de uma Matriz de Correlação Linear de Pearson entre o Potencial Hídrico obtido no potencial de base, Potencial Hídrico obtido após o meio-dia e Precipitação Pluviométrica mensal a fim de verificar o grau de correlação Linear entre estas variáveis. Num segundo momento foi realizada uma análise Multivariada, atarvés do Teste de Hotteling (aproximação de F) de comparação das médias dos potenciais hídricos (potencial de base e após o meio-dia) obtidos entre Neea macrophylla e Cecropia palmata. Da mesma forma foi verificado o efeito do período climático sobre tais Potenciais Hídricos de cada uma das espécies potencialmente acumuladoras de P também a partir da aplicação do Teste de Hotteling. O programa estatístico utilizado foi o BioEstat 3.0 (Ayres et al, 2003).

\section{RESULTADOS E DISCUSSÕES}

O efeito da agregação das árvores de Neea macrophylla acerca das radiaçôes médias (w.m ${ }^{-2} \cdot \mathrm{nm}^{-1}$ ) Fotossinteticamente Ativa (PAR) e Ativa na Regiāo do Azul (BAR) abaixo das copas de Neea macrophylla onde o agregado com o menor número de indivíduos (terceiro agregado) apresentou os maiores valores de PAR e BAR sugerindo que uma agregação mais densa já reduz quantitativamente a luz sob a projeção das copas sobretudo ao nível do solo, aumentando gradativamente até $1,80 \mathrm{~m}$ conforme FIGURA 1. Já a radiação PAR e BAR abaixo das copas de Cecropia palmata não diferiram entre os 3 indivíduos estudados, independente do número de folhas que possuíam entretanto, ao nível do solo as 2 radiações foram maiores do que a 1,80m conforme FIGURA 2.

Quanto à PAR e BAR Totais $\left(\mathrm{w} / \mathrm{m}^{2}\right)$ observamos que Cecropia palmata permitiu uma passagem maior destas radiações abaixo de suas copas fator este diretamente relacionado à arquitetura da copa da espécie conforme FIGURAS 3, 4 e 5. Como possíveis componentes arbóreos no Enriquecimento de Capoeiras e em Sistemas Agroflorestais tais variações na PAR e BAR abaixo das copas podem ser decisivas nos processos fotossintéticos e de morfogênese sobre as espécies a serem consorciadas.

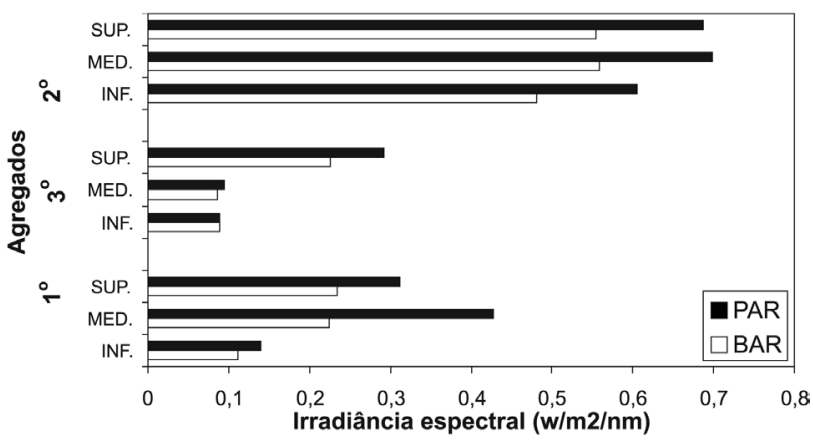

Figura 1- Irradiância espectral média $\left(w \cdot m^{-2} \cdot \mathrm{nm}^{-1}\right)$ nos intervalos de 400 $500 \mathrm{~nm}$ (BAR) e 400-700 nm (PAR) sob as copas de três agregados de Neea macrophylla ( $10=$ nove indivíduos; $20=$ cinco indivíduos e $30=$ um indivíduo) e em três estratos (inferior $=I N F$, médio $=$ MED e superior $=S U P$ ).

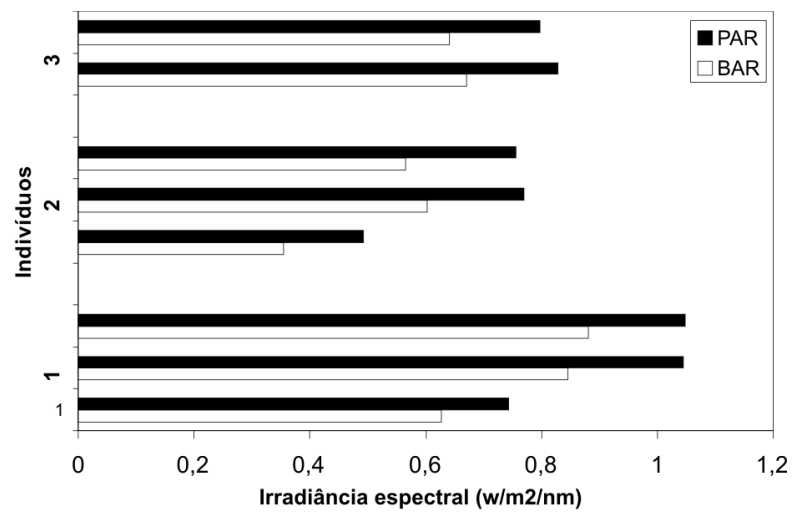

Figura 2 - Irradiância espectral média $\left(w^{\prime} \cdot m^{-2} \cdot \mathrm{nm}^{-1}\right)$ sob as copas de três indivíduos de Cecropia palmata $(10=$ com 9 folhas; $20=$ com 12 folhas e $30=$ com 8 folhas) e em 3 extratos (inferior $=$ na altura do solo; médio $=a$ $80 \mathrm{~cm}$ e superior $=\mathrm{a} 1,80 \mathrm{~m})$. 


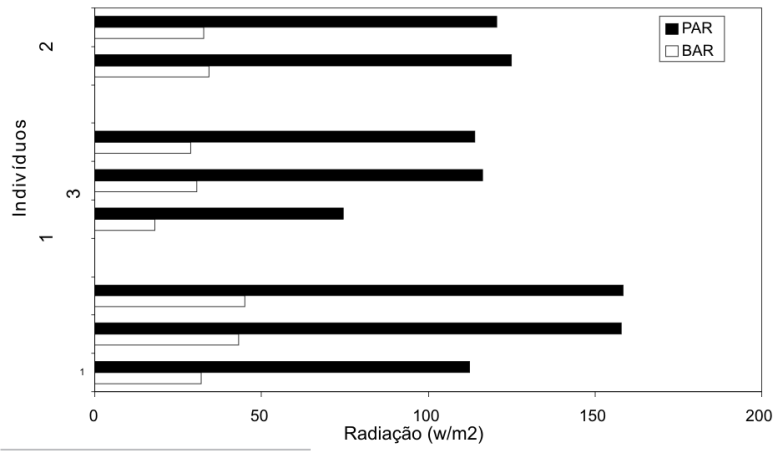

Figura 3 -Total das Radiações Fotossinteticamente ativa (PAR) e Ativa na Região do Azul (BAR) medidas abaixo das copas de Cecropia palmata $(10=$ com 9 folhas; $20=$ com 12 folhas e $30=$ com 8 folhas) e em 3 estratos distintos.

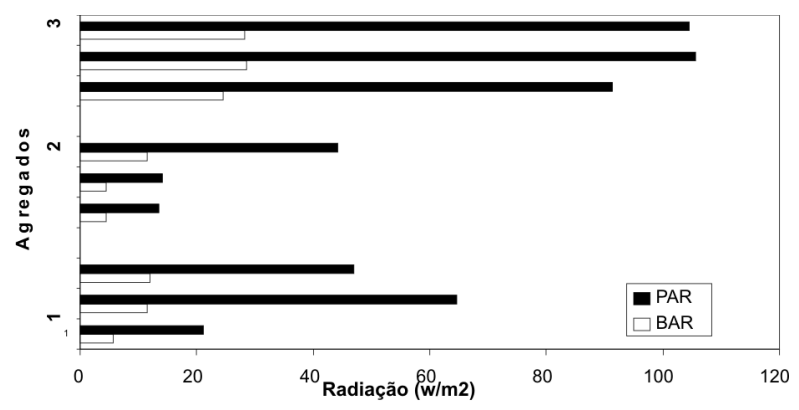

Figura 4 - Total das Radiações Fotossinteticamente Ativa (PAR) e Ativa na Região do Azul (BAR) medidas abaixo das copas de 3 agregados de Neea macrophylla $(10=$ nove indivíduos; $20=$ cinco indivíduos e $30=$ um indivíduo).

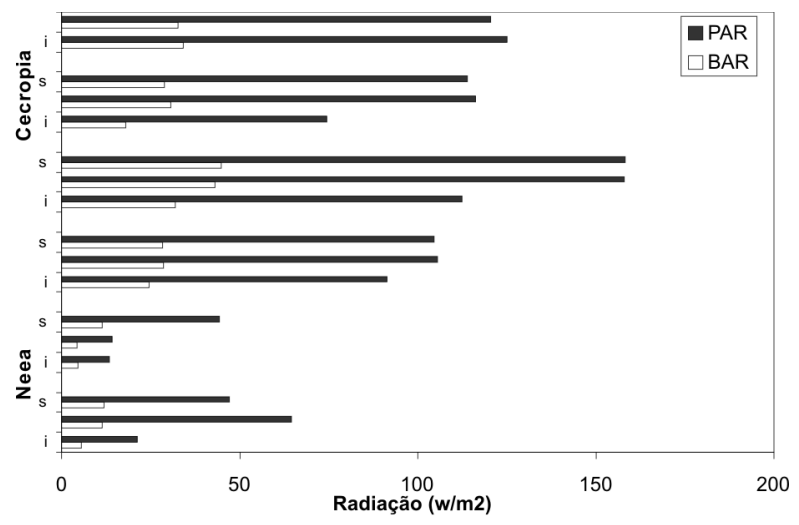

Figura 5 - Total das radiações Fotossinteticamente Ativa (PAR) e Ativa na Região do Azul (BAR) abaixo das copas de 3 agregados de Neea macrophylla $(10=$ nove indivíduos; $20=$ cinco indivíduos e $30=$ um indivíduo $)$ e de 3 indivíduos de Cecropia palmata $(10=\operatorname{com} 9$ folhas; $20=$ com 12 folhas e $30=$ com 8 folhas), em 3 distintos estratos ( $i=$ inferior, $m=$ medio e $\mathrm{s}=$ superior).
Os resultados demonstraram que a espécie Cecropia palmata apresentou valores muito próximos da Irradiância Espectral acima das copas (conforme FIGURA 7) e que pouco bloqueio ofereceu à passagem de luz. É uma espécie portanto que pode ser adicionada à Sistemas Agroflorestais sem qualquer restrição do fator luz para espécies associadas. Quanto à razão vermelho:vermelho extremo, esta foi maior abaixo das copas de Cecropia palmata do que abaixo das copas de Neea macrophylla com probabilidade $P=O$ de que isto se deva ao acaso conforme FIGURA 6. Tais valores foram um pouco maiores do que os encontrados por Sá et al (1999) em vegetação secundária com seis anos de regeneração na mesma região.

A Análise Multivariada a partir da aplicação do Teste de Hotteling (TABELA 1) revelou diferença significativa através da análise conjunta das três variáveis estudadas (PAR, BAR e PhyAR) entre as duas espécies acumuladoras de P, Neea macrophylla e Cecropia palmata. A arquitetura de copa de Cecropia palmata propiciou maior radiação total tanto de BAR, PAR quanto de PhyAR, sugerindo que o ambiente luminoso criado a partir de um plantio sistemático de Cecropia palmata pouco restringe as radiaçóes acima citadas e que portanto condições favoráveis aos processos fotossintéticos e de morfogênese estarão garantidas às espécies companheiras que vierem a se localizar sob influência das copas consideradas.

Quanto à caracterização do Potencial Hídrico em espécies potencialmente acumuladoras de $\mathrm{P}$, esta demonstrou uma variação onde Cecropia palmata conseguiu manter Potenciais Hídricos menos negativos do que Neea macrophylla e Casearea arborea conforme FIGURAS 8, 9 e 10. Estes resultados sugerem que C.palmata apresentou uma vantagem em relação às demais espécies sob condiçôes de déficit hídrico. Casearea

Tabela 1 - Análise Multivariada através da aplicação do Teste de Hotteling de comparação das três radiações em w/m² , BAR, PAR e PhyAR abaixo das copas de duas espécies acumuladoras de P, Neea macrophylla e Cecropia palmata em floresta secundária (sito I) com aproximadamente 6 anos de regeneração, Pará, Brasil.

\begin{tabular}{llll}
\hline & BAR & PAR & PhyAR \\
\hline Amostra 1:Neea & 13.1472 & 50.1612 & 1.0067 \\
\hline Variância $=$ & 99.3913 & 1462.9194 & 0.1775 \\
\hline Tamanho = & 24 & 24 & 24 \\
\hline Amostra 2: Cecropia & 33.0563 & 122.3304 & 1.2975 \\
Variância = & 176.251 & 2243.8806 & 0.0283 \\
\hline Tamanho = & 24 & 24 & 24 \\
\hline T2 (Hotteling) = & 34.8312 & --- & --- \\
\hline F $=$ & 11.1056 & --- & --- \\
\hline Graus de liberdade & 3,44 & --- & --- \\
(p) $=$ & 0,0001 & --- & --- \\
\hline
\end{tabular}




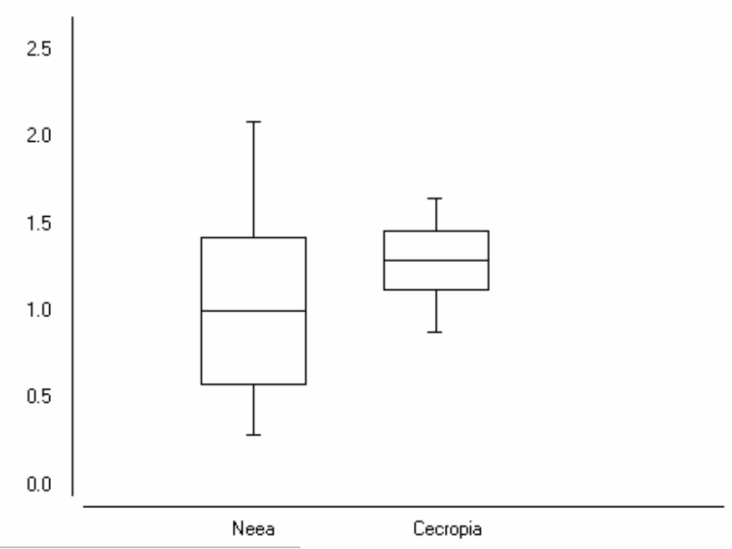

Figura 6 - Razão vermelho:vermelho extremo abaixo das copas de Neea macrophylla e Cecropia palmata em floresta secundária à Nordeste do Estado do Pará.*

"médias diferentes significativamente ao nível de 1\% de probabilidade

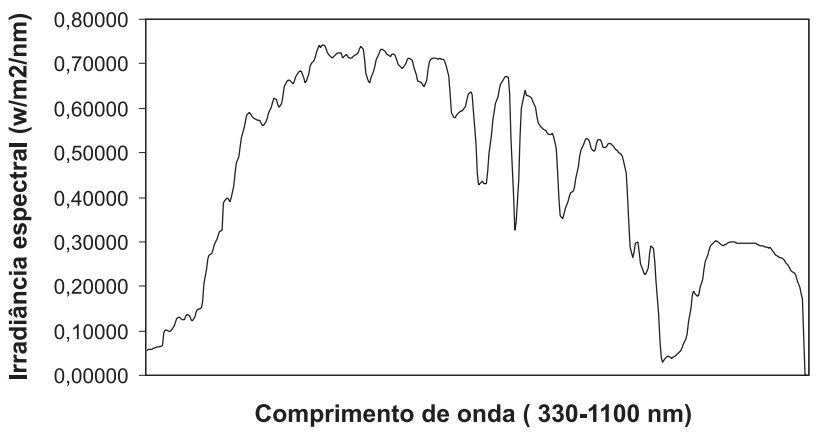

FIGURA 7- Irradiância Espectral (w/m2/nm) no intervalo de 330 a 1100 nm acima das copas das espécies estudadas, no dia 16 de janeiro de 2003, Igarapé-Açú, Pará, Brasil.

arboria foi a espécie que desenvolveu potenciais hídricos mais negativos chegando à $-2,74 \mathrm{MPa}$ após o meio-dia sugerindo uma certa sensibilidade da espécie a períodos com baixa precipitação pluviométrica como o estudado.

Quando analisa-se os dados relativos ao gradiente de potencial hídrico $\left(\Delta \Psi=\Psi_{\text {apossomeio-dia }}-\Psi_{\text {potenncial de basse }}\right)$ das 3 espécies estudadas como forma de caracterizar a elasticidade que tem este parâmetro sob diferentes condições pluviométricas, notase que os maiores $\Delta \Psi$ foram obtidos por Casearea arborea em todos os meses estudados de acordo com a FIGURA 11, enquanto Cecropia palmata obteve os mais baixos $\Delta \Psi$, sugerindo que Casearea apresenta maior elasticidade, isto é, leva mais tempo para recuperar o status hídrico pois atinge valores muito negativos ao meio-dia, o que já não acontece com Cecropia palmata que consegue manter Potenciais Hídricos menos negativos tornando-se mais estável ao longo do dia. Neea macrophylla pode ser considerada espécie de comportamento intermediário porém mais similar à Casearia do que à Cecropia, ou seja mais sensível ao período seco.

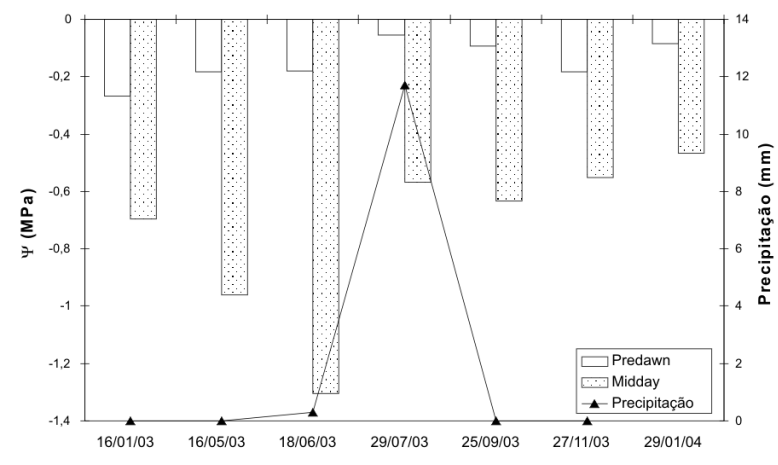

Figura 8 - Comportamento do Potencial Hídrico ( $\Psi)$ em MPa de Cecropia palmata sob baixa precipitação pluvimétrica ( $\mathrm{mm} / \mathrm{dia})$ em floresta secundária, Pará, Brasil.

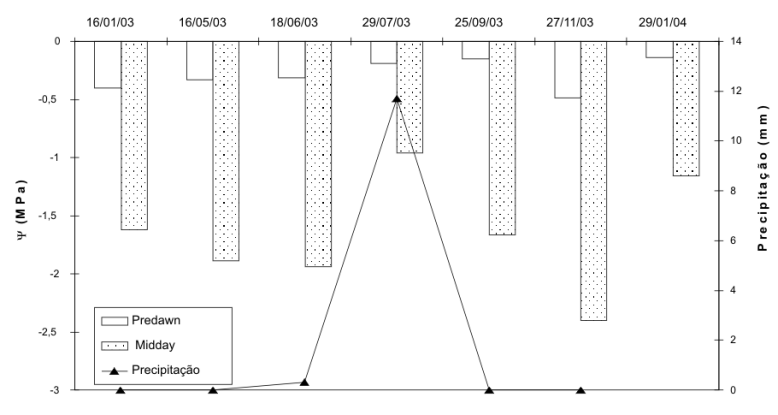

Figura 9 - Potencial Hídrico ( $\Psi$ ) em MPa de árvores de Neea macrophylla, no período de janeiro de 2003 à janeiro de 2004 sob baixa precipitação pluviométrica (mm/dia) em floresta secundária, Igarapé-Açú, Pará, Brasil.

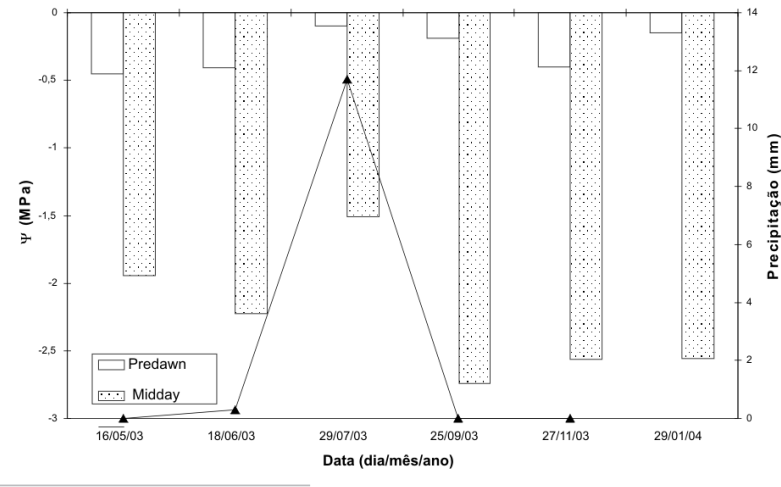

Figura 10- Comportamento do Potencial Hídrico $(\Psi)$ em MPa de árvores de Casearea arbórea sob baixa precipitação pluviométrica (mm/dia)

A construção da Matriz de Correlação (TABELA 2) revelou correlação positiva $(\mathrm{r}=0,6425)$ entre o potencial hídrico de base e após o meio-dia com probabilidade $(P)$ igual a 0,0054 de que isto se deva ao acaso. À medida que diminuia o potencial hídrico de base, o obtido após o meio-dia também tendia a diminuir . 
Tabela 2 - Matriz de Correlação Linear de Pearson entre o potencial de base (coluna 1), potencial hídrico após o meio-dia (col. 2) e precipitação mensal (col.3) observadas em folhas de Neea macrophylla, Cecropia palmata e Casearia arborea em floresta secundária com 6 anos de regeneração.

\begin{tabular}{|c|c|c|c|}
\hline & $\begin{array}{l}\text { Colunas } \\
1 \text { e } 2\end{array}$ & $\begin{array}{l}\text { Colunas } \\
1 \text { e } 3\end{array}$ & $\begin{array}{l}\text { Colunas } \\
2 \text { e } 3\end{array}$ \\
\hline $\mathrm{n}$ (pares) $=$ & 17 & 17 & 17 \\
\hline$r($ Pearson $)=$ & 0.6425 & -0.0729 & 0.2602 \\
\hline IC $95 \%=$ & 0.23 a 0.86 & -0.53 a 0.42 & -0.25 a 0.66 \\
\hline IC $99 \%=$ & 0.07 a 0.90 & -0.64 a 0.55 & -0.40 a 0.74 \\
\hline $\mathrm{R} 2=$ & 0.4128 & 0.0053 & 0.0677 \\
\hline$t=$ & 3.2475 & -0.2829 & 1.0439 \\
\hline $\mathrm{GL}=$ & 15 & 15 & 15 \\
\hline$(p)=$ & 0.0054 & 0.7811 & 0.313 \\
\hline Matriz de Correlação & Coluna 1 & Coluna 2 & Coluna 3 \\
\hline Coluna $1=\Psi$ predawn & 1 & --- & --- \\
\hline Coluna $2=\Psi$ midday & 0.6425 & 1 & --- \\
\hline Coluna $3=$ precipitation & -0.0729 & 0.2602 & 1 \\
\hline
\end{tabular}

A Análise Multivariada a partir da aplicação do Teste de Hotteling demonstrou que folhas de Cecropia palmata e Neea macrophylla, ambas espécies acumuladoras de $\mathrm{P}$, diferiram significativamente ao nível de $1 \%$ de probabilidade através da análise conjunta de duas variáveis, potencial hídrico de base e após o meio-dia, onde os tecidos de Cecropia palmata mantiveram-se mais túrgidos do que os de Neea $(-1,74 \mathrm{MPa})$ ao meio dia conforme TABELA 3. Da mesma forma, Cecropia diferiu de Casearia ao nível de 1\% de probabilidade onde a tolerância de Casearia arborea pode ser evidenciada a partir da determinação de potencial hídrico igual a -2,19 MPa em suas folhas ao meio-dia, um valor extremamente negativo sugerindo resistência ao déficit hídrico temporário na região.

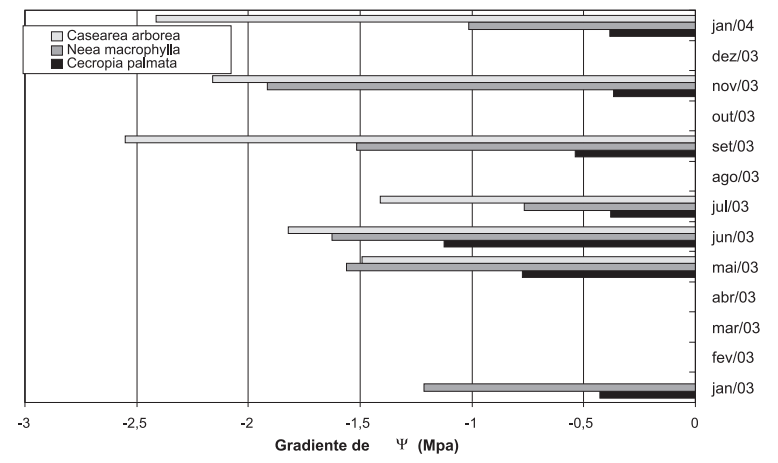

Figura 11- Gradiente de Potencial Hídrico $\left(\Delta \Psi=\Psi_{\text {após o meio-dia }}-\Psi_{\text {potencial de }}\right.$ base) de Cecropia palmata, Neea macrophylla e Casearea arborea ao longo de 1 ano de observação em vegetação secundária em estágio inicial de regeneração,Pará, Brasil.
Tabela 3 - Análise Multivariada, através da aplicação do Teste de Hotelling de comparação de 2 conjuntos de variáveis, sendo a variável 1 o potencial hídrico obtido no potencial de base e a variável 2 o potencial hídrico obtido após o meio-dia entre folhas de Cecropia palmata e Neea macrophylla, Cecropia e Casearia e Neea e Casearia.

\begin{tabular}{|c|c|c|}
\hline Cecropia X Neea & $\begin{array}{l}\text { Var } 1 \\
\text { ( } \Psi \text { de base) }\end{array}$ & $\begin{array}{l}\text { Var } 2 \\
\text { ( } \Psi \text { após o meio-dia) }\end{array}$ \\
\hline Amostra 1: Cecropia palmata & -0.16 & -0.785 \\
\hline variância = & 0.0058 & 0.087 \\
\hline Amostra 2: Neea macrophylla & -0.3124 & -1.7455 \\
\hline variância $=$ & 0.0158 & 0.2258 \\
\hline T2 (Hotteling) = & 17.6975 & \\
\hline$(p)=$ & 0.0103 & --- \\
\hline \multicolumn{3}{|l|}{ Cecropia x Casearia } \\
\hline Amostra 1: Cecropia palmata & -0.16 & -0.785 \\
\hline variância = & 0.0058 & 0.087 \\
\hline Amostra 2: Casearia arborea & -0.3103 & -2.1973 \\
\hline variância = & 0.0241 & 0.243 \\
\hline T2 (Hotteling) = & 35.0935 & --- \\
\hline$(p)=$ & 0.0021 & --- \\
\hline \multicolumn{3}{|l|}{ Neea x Casearia } \\
\hline Amostra 1: Neea macrophylla & -0.3124 & -1.7455 \\
\hline variância = & 0.0158 & 0.2258 \\
\hline Amostra 2: Casearia arborea & -0.3103 & -2.1973 \\
\hline variância = & 0.0241 & 0.243 \\
\hline T2 (Hotteling) = & 3.1457 & \\
\hline$(p)=$ & 0.3015 & --- \\
\hline
\end{tabular}

Contudo, não houveram diferenças significativas entre os potenciais hídricos de Neea e Casearia.

O efeito do período climático sobre os potenciais hídricos das folhas de Cecropia palmata puderam ser observados entre Janeiro de 2003 (período chuvoso) e Junho de 2003 (período de estiagem), respectivamente $232,2 \mathrm{~mm}$ e $61,8 \mathrm{~mm}$ de precipitação de acordo com a TABELA 4. O período com a menor precipitação pluviométrica resultou em potenciais hídricos mais negativos sobretudo após o meio-dia. Da mesma forma houve diferenças significativas entre os meses de Janeiro de 2003 e Setembro de 2003 (período seco), respectivamente 232,2 e $45 \mathrm{~mm}$ de precipitação. Comportamento hídrico similar foi observado em Neea macrophylla. De forma geral, ao longo de 1 ano de observação quando levou-se em consideração as 3 especies estudadas, não houveram correlaçôes positivas entre potencias hídricos no predaw e no midday (FIGURA 12). 
Tabela 4- Análise Multivariada, através da aplicação do Teste de Hotelling para comparação de 2 conjuntos de variáveis, sendo a variável 10 potencial hídrico obtido no potencial de base e a variável 2 o Potencial Hídrico obtido após o meio-dia em folhas de Cecropia palmata e Neea macrophylla entre 0 período chuvoso (janeiro $=232,2 \mathrm{~mm}$ chuva) e o período de estiagem (junho $=$ $61,8 \mathrm{~mm})$ e entre o período chuvoso e seco $(45 \mathrm{~mm})$.

\begin{tabular}{|c|c|c|}
\hline $\begin{array}{l}\text { Cecropia palmata } \\
\text { Período chuvoso X Período de estiagem }\end{array}$ & $\begin{array}{l}\text { Var } 1 \\
\text { (potencial de } \\
\text { base) }\end{array}$ & $\begin{array}{l}\text { Var } 2 \\
\text { (após o } \\
\text { meio-dia) }\end{array}$ \\
\hline Amostra 1: JANEIRO DE 2003 & -0.2679 & -0.6949 \\
\hline Amostra 2: JUNHO DE 2003 & -0.18 & -1.305 \\
\hline T2 (Hotteling) = & 58.4301 & --- \\
\hline$(p)=$ & 0.0009 & \\
\hline \multicolumn{3}{|l|}{ Período Chuvoso X Período Seco } \\
\hline Amostra 1: JANEIRO / 2003 & -0.2679 & -0.6949 \\
\hline Amostra 2: SETEMBR0/2003 & -0.0917 & -0.6333 \\
\hline T2 (Hotteling) = & 48.8895 & \\
\hline$(p)=$ & 0.0007 & --- \\
\hline \multicolumn{3}{|l|}{$\begin{array}{l}\text { Neea macrophylla } \\
\text { Período chuvoso X Período de estiagem }\end{array}$} \\
\hline Amostra 1: JANEIR0/2003 $(232,2 \mathrm{~mm})$ & -0.4043 & -1.6196 \\
\hline Amostra 2: JUNHO /2003 ( 61,8mm) & -0.315 & -1.94 \\
\hline T2 (Hotteling) $=$ & 10.1573 & \\
\hline$(p)=$ & 0.0223 & --- \\
\hline \multicolumn{3}{|l|}{ Período Chuvoso X Período Seco } \\
\hline Amostra 1: JANEIRO/2003 (232,2 mm) & -0.4043 & -1.6196 \\
\hline Amostra 2: SETEMBR0/2003 (45 mm) & -0.15 & -1.665 \\
\hline T2 (Hotteling) = & 32.6497 & \\
\hline$(p)=$ & 0.0007 & \\
\hline
\end{tabular}

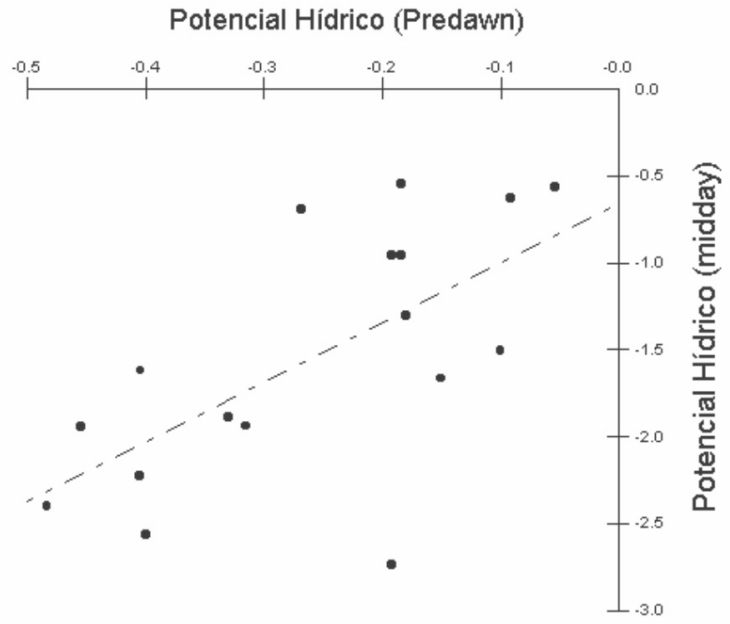

Figura 12 - Correlação Linear $(r=0,64) \operatorname{com} P=0,0054$ entre Potencial Hídrico ( $\Psi$ ) em MPa, observado em folhas de Neea macrophylla, Cecropia palmata e Casearia arborea no potencial de base ( 5:00 h) e após o meio-dia (12:00 h) ao longo de 1 ano.

\section{CONCLUSÕES}

A densidade de agregação das árvores teve maior influência na radiação que atravessava as copas de Neea macrophylla do que às de Cecropia palmata. Neea macrophylla e Cecropia palmata, espécies potencialmente acumuladores de P disponibilizaram abaixo de suas copas, Radiação Fotossinteticamente Ativa, Radiação Ativa na região do Azul e Radiação Ativa ao Fitocromo diferentes quantitativamente entre si em função de distintas arquiteturas de copa. Árvores de Cecropia palmata restringiram menos radiação do que Neea macrophylla sugerindo melhores condiçōes aos processos fotossintéticos e morfogênicos às espécies sob influência dessas copas. As flutuações na precipitação ao longo do ano de 2003 determinaram flutuações nos estados de energia potencial da água tanto no potencial de base quanto após o meio-dia em tecidos foliares de Neea macrophylla e Cecropia palmata, indicando que Cecropia palmata deve ser mais tolerante ao déficit hídrico porque manteve os Potenciais Hídricos maiores enquanto Neea macrophylla e Casearia arborea foram mais sensíveis.

\section{AGRADECIMENTOS \\ PROJETO SHIFT \& PROJETO LBA}

\section{BIBLIOGRAFIA CITADA}

Ayres, M.; Ayres Junior, Ayres, D.L. \& dos Santos,A .S.- BioEstat 3.0. Aplicaçôes estatísticas nas áreas das Ciências Biológicas e Médicas. Sociedade Civil Mamirauá/MCT-CNPQ/ Conservation International, Belém, Pará, 2003.

Carswell, F.E.; Meir, P.; Wandelli, E.V.; Bonates, L.C.M.; Kruijt, B.; Barbosa, E.M.; Nobre, A.D.; Grace, J. e Jarvis, P.G.Photosynthetic Capacity in a Central Amazonian rain Forest. Tree Physiology, Heron Publishing, Canadá, 2000, 20: 179:186.

Carvalho, Cláudio José Reis de. Respostas de plantas de Schizolobium amazonicum [S. parahyba var. amazonicum] e Schizolobium parahyba [Schizolobium parahybum] à deficiência hídrica. Rev. Arvore, nov./dez. 2005, vol.29, no.6, p.907-914

Kull O., Broadmeadow, M., Kruijt, B. e Meir, P. - Light distribution and foliage Structure in an oak canopy. Trees, 1999, 14:2, pp. 55-64.

Meinzer, F.C., Goldstein, G. e Andrade, J.L.- Regulation of water flux through tropical forest canopy trees: Do universal rules apply ? Tree Physiology, Canadá, 2001, 21:19 - 26.

Rigamonte-Azevedo, Onofra Cleuza, WADT, Paulo Guilherme Salvador e WADT, Lúcia Helena de Oliveira. Potencial de produção de óleo-resina de copaíba (Copaifera spp) de populaçōes naturais do sudoeste da Amazônia. Rev. Arvore, jul./ago. 2006, vol.30, no.4, p.583-591. 
Sá, T. D. de A; De Oliveira, V.C.; De Araújo, A.C. e Brienza Júnior, S.- Spectral Irradiance and stomatal conductance of enriched fallows with fast-growing trees in eastern Amazonia, Brazil. Agroforestry Systems, Kluwer Academic Publishers.1999, 47: 289-303.

Sellin, Arne- Morphological and stomatal responses of Norway spruce foliage to irradiance within a canopy depending on shoot age. Enviromental and Experimental Botany, 2001, 45:2, pp. 115-131.

Recebido em 30/06/2007

Aceito em 10/03/2008 